\title{
Simulação de produtividade futura de soja em Piracicaba-SP com base em projeções de mudanças climáticas
}

\author{
Evandro Henrique Figueiredo Moura da Silva ${ }^{1}$, Rodolfo Armando de Almeida Pereira ${ }^{2}$, Alexandre Ortega Gonçalves ${ }^{3}$, \\ Álex Júnior Zanchet Bordignon ${ }^{4}$, Fábio Ricardo $\operatorname{Marin}^{5^{(*)}}$ \\ ${ }^{1}$ Mestrando em Engenharia de Sistemas Agrícolas, ESALQ/USP, Piracicaba, São Paulo, ehfmsilva@usp.br \\ ${ }^{2}$ Mestrando em Engenharia de Sistemas Agrícolas, ESALQ/USP, Piracicaba, São Paulo, rodolfopereira@usp.br \\ ${ }^{3}$ Doutorando em Engenharia de Sistemas Agrícolas, ESALQ/USP, Piracicaba, São Paulo, alexandre.ortega@embrapa.br \\ ${ }^{4}$ Graduando em Engenharia Agronômica, ESALQ/USP, Piracicaba, São Paulo, alex.bordignon@usp.br \\ ${ }^{5}$ Professor Associado do Departamento de Engenharia de Biossistemas, ESALQ/USP, Piracicaba, São Paulo, fabio.marin@usp.br \\ ${ }^{(*)}$ Autor para correspondência
}

\section{INFORMAÇÕES}

História do artigo:

Recebido em 16 de Junho de 2017

Aceito em 10 de agosto de 2017

Termos para indexação:

modelos de culturas

DSSAT

MAGé

Glycine max

\section{RESUMO}

O Brasil é um dos maiores países produtores de soja (Glycine max L.). Essa planta é a principal fonte de proteína alimentar do planeta. Com base na importância econômica e social da soja no contexto mundial, é importante considerar as projeções futuras da produção dessa oleaginosa dentro do debate internacional de mudanças climáticas; e previsão do aumento populacional global, de 2 bilhões de habitantes, até o ano de 2050. Assim, pretende-se avaliar o impacto das mudanças climáticas sobre o desenvolvimento da cultura da soja na região de Piracicaba-SP utilizando dois modelos o DSSAT/CROPGRO Soybean e o MAGé adaptado. O modelo DSSAT/CROPGRO foi calibrado para a região do estudo com dados de clima, solo e dados biométricos de soja (BRS 399RR) obtidos experimentalmente. Os cenários de mudanças climáticos do IPCC selecionados nesse estudo foram RCP4.5 (2020-2050), RCP4.5 (2020-2050), RPP8.5 (2050-2080) e RCP8.5 (2050-2080). Observou-se que o modelo DSSAT/ CROPGRO apresentou uma boa calibração, representando bem a produção atual de soja na região. Com relação às simulações em cenários futuros houve des créscimo da produtividade de soja na região quando somente o fator temperatura foi considerado e aumento da produtividade quando o incremento na $\left[\mathrm{CO}_{2}\right]$ foi considerado no modelo. Nessa segunda perspectiva, os cenários de mudanças climáticas seriam benéficos a produção de soja em Piracicaba. Já o modelo MAGé adaptado mostrou-se produtividades superestimadas em todas as situações estudadas.

(c) 2017 SBAgro. Todos os direitos reservados.

\section{Introdução}

A soja (Glycine max L.) é uma planta oleaginosa que se destaca por ser uma das principais culturas agrícolas, sendo a principal geradora de divisas brasileiras e a principal fonte de proteína alimentar do planeta (SILVA, 2014; MA-
TOS et al, 2015; CONAB, 2016). Segundo dados da United States Department of Agriculture (2016) o farelo de soja representou, nas últimas cinco safras, o equivalente a $68,81 \%$ de toda a proteína de origem vegetal produzida mundialmente. Essa produção proteica procede de mais de 120 milhões de hectares agrícolas, dos quais mais de 33 milhões de hec- 
tares estão em território brasileiro (USDA, 2017).

Com base na relevância da soja no contexto mundial, é importante considerar as projeções futuras da produção dessa oleaginosa dentro do debate internacional de mudanças climáticas. Tendo em vista a previsão do aumento populacional global, como também relatado Foley (2011) deverá ser de 3 bilhões de habitantes, até o ano de 2050 esse debate torna-se ainda mais relevante.

A modelagem de sistemas agrícolas tem contribuído para a simulação de produtividade de culturas agrícolas. Esses modelos, segundo Rosenzweig et al (2013), são cientificamente aceitos para analisar os impactos da mudança climática na agricultura. Os modelos, por definição, são essencialmente teóricos e derivam de um conjunto de teoremas formulados a partir de premissas ou postulados (ALPHA e CHIANG, 2005). As teorias, por definição, são abstrações do mundo real, nesse sentido buscam estreitar problemas complexos (POPPER, 2005), sendo, portanto, um recurso encontrado para isolar fatores e relações essenciais de uma complexa realidade: o crescimento vegetal e sua interação com o meio que o cerca, por exemplo. O grau de complexidade de cada modelo depende principalmente da abordagem e dos problemas que os modeladores buscam resolver. Segundo Jones, Keating e Porter (2001), os modelos complexos baseados em processos geralmente contam com estruturas modulares específicas.

O presente estudo tem como objetivo comparar dois modelos com diferentes graus de complexidade o DSSAT/ CROPGRO Soybean e o MAGé adaptado, para a simulação de dados de produtividade da cultura da soja atual e futura para a região de Piracicaba - SP.

\section{Material e métodos}

Apresenta-se nessa seção o material e métodos do presente estudo, que será apresentado nos diferentes tópicos: (i) instalação do experimento de campo e coleta de dados experimentais; (ii) calibração do modelo Decision Support System for Agrotechnology Transfer (DSSAT/CROPGRO Soybean) pra a região de Piracicaba-SP; (iii) indicadores de eficiência de modelagem; (iv) execução do modelo genérico para culturas agrícolas e (iv) metodologia dos cenários futuros de mudanças climáticas.

\section{Experimento de campo e o Modelo DSSAT/CROPGRO Soybean}

O experimento foi conduzido no município de Piracicaba - SP, na área experimental do Departamento de Engenharia de Biossistemas da Escola Superior de Agricultura “Luiz de Queiroz" (ESALQ/USP), latitude 2242’32”S, longitude 4737'45"O e altitude de $548 \mathrm{~m}$, adjacente ao Posto Meteorológico de Piracicaba (PME). Buscou-se conduzir uma lavoura de soja, em condições ideais de manejo com o objetivo de se aproximar ao conceito de produtividade potencial. A qual segundo Marin (2014), é a produtividade que pode ser obtida por um genótipo adaptado sob con€ dições ótimas de cultivo, isso é: sem limitações hídricas e sem a intervenção de fatores redutores (pragas, doenças e nutrientes). Assim, como estratégia de obtenção de dados próximos a produtividade potencial a cultura foi instalada em área com sistema de irrigação (controlado pelo balanço hídrico) por gotejamento, por meio de gotejadores autocompensantes (PCJ, Netafin, 8 L.h ${ }^{-1}$ ) e um rigoroso monitoramento de pragas e doenças, com pulverizações químicas frequentes e um bom manejo nutricional com base na análise química do solo.

Elencou-se uma cultivar recomendada para a região, a BRS 399-RR (EMBRAPA, 2016). Outra preocupação tomada com a escolha do material genético era que este representasse as características agronômicas adotadas na agricultura brasileira como: (i) precocidade do ciclo (grupo de maturação 6); (ii) resistência ao herbicida glifosato; (iii) resistência e/ou tolerância a doenças (cancro da haste, oídio, podridão parda da haste, podridão radicular) e nematoides (nematoide da galha e do cisto).

Coletou-se de maneira aleatória 10 plantas a cada medição biométrica, ao todo, foram feitas sete coletas ao longo do ciclo da cultura da soja, nos DAP (dias após o plantio): $29,39,46,53,60,78$ e 94 . As coletas de campo geraram os dados de entrada para a calibração do modelo: (a) área foliar específica, (b) biomassa foliar, (c) índice de área foliar, (d) biomassa de caule, (c) biomassa vegetativa e (e) massa seca de grãos, produtividade final. Os dados meteorológicos de entrada foram obtidos no PME, para o período dos últimos 30 anos (1986-2016): (i) temperatura máxima; (ii) temperatura mínima, radiação global e precipitação ao longo de 30 anos do PME.

Construiu-se um arquivo para o solo da área experimental, o Nitossolo Vermelho Eutroférrico latossólico. Para isso levantou-se os dados coletados no local, pela equipe do Prof. Paulo Leonel Libardi, ainda não publicados: (a) densidade global do solo (SBDM, do inglês soil bulk density); (b) ponto de murcha permanente (SLLL, do inglês soil lower limit); (c) capacidade de campo (SDUL, do inglês soil drained upper limit); (d) ponto de saturação (SSAT, do inglês soil saturated); (e) condutividade hidráulica saturada (SSKS, do inglês soil saturated hydraulic condutivity); (f) teor de carbono orgânico (SLOC, do inglês organic carbon) e (g) teor de argila (SLCL, do inglês clay) e teor de silte (SLSI, do inglês silt).

Com os dados de solo, clima e as biometrias realizadas buscou-se calibrar o modelo DSSAT/CROPGRO Soybean para a região de Piracicaba - SP, seguindo o procedimento proposto por Marin et al. (2015) em conjunto com o procedimento de calibração automática (para ajuste fino de pa- 
râmetros) conforme descrito em Marin et al. (2011). Foram estudados e alterados (quando necessário) os seguintes parâmetros para o ajuste da curva: (a) SLPF (do inglês, photosynthesis fator) que é um fator relacionado a fertilidade do solo capaz de descrever possíveis reduções na fotossíntese devido a deficiência de nutrientes no solo; (b) Comprimento crítico do dia curto (CSDL, do inglês critical short day length); (b) a resposta relativa do desenvolvimento da planta ao fotoperíodo (PPSEN, do inglês relative response of development to photoperiod); (c) o tempo entre a emergência e o início do florescimento da planta, (EM-FL, do inglês time between plant emergence and flower appearance - R1); (d) tempo entre o estádio R1 e o início da frutificação (FL-SH, do inglês time between first flower and first pod - R3); (e) tempo entre R1 e o início da formação da semente (FL-SD, do inglês time between first flower and first seed - R5); (f) tempo entre R5 e a maturidade fisiológica da planta (SD-PM, do inglês time between first seed and physiological maturity - R7); (g) tempo entre R1 e o fim da expansão foliar (FL-LF, do inglês time between first flower and end of leaf expansion); (h) taxa máxima de fotossíntese (LFMAX, do inglês maximum leaf photosynthesis rate); (i) área foliar específica (SLAVR, do inglês specific leaf area); (j) duração do enchimento de grãos (SFDUR, do inglês seed filling duration); (1) número médio de sementes por vagem (SDPDV, do inglês average seed per pod under); $(\mathrm{m})$ tempo requerido para que a cultivar alcance a máxima carga de vagens (PODUR, do inglês time required for cultivar to reach final pod load); (n) índice de colheita (THRSH, do inglês threshing percentage); (o) máximo peso por semente (WTPSD, do inglês Maximum weight per seed).

\section{Modelo Agrometeorológico Genérico de Produção Vegetal (MAGé)}

Vianna et al. (2017) desenvolveram um modelo mecanístico, o MAGé, caracterizando o crescimento e desenvolvimento da planta utilizando se de equações matemáticas pata tal. O MAGé é capaz de estimar a produtividade potencial e atingível considerando variáveis meteorológicas de entrada comumente medidas, como a temperatura do ar e precipitação. Esse modelo correlaciona parâmetros vegetativos e fisiológicos com as variáveis meteorológicas. 0 índice de área foliar (IAF), coeficiente de extinção (k), índice de colheita (IC), umidade de grão (u) e eficiência do uso da radiação (RUE) servem para descrever as características da planta de maneira numérica e sucinta.

O experimento à campo possibilitou ampliar a gama de variáveis meteorológicas de entrada, assim foram mensurados a radiação global (Qg), temperatura do ar (Tar) e precipitação (P). Consequentemente se fez necessário adaptar o MAGé para a entrada de valores de Qg mensurados e também em detrimento da característica da soja, que é sensível ao fotoperíodo. Nesse sentido a produção diária em $\mathrm{g} / \mathrm{m}^{2}$ é obtida por:

$$
\mathrm{PP}_{\left(\mathrm{g} / \mathrm{m}^{2}\right)}=\frac{\mathrm{aPAR} \cdot \text { Rue } \cdot \mathrm{IC} \cdot \mathrm{IAF} \cdot \mathrm{Ft} \cdot \mathrm{Fcd}}{(1-u)}
$$

em que aPAR é a radiação absorvida pela cultura, dado em $\mathrm{MJ} \cdot \mathrm{m}^{-2} \cdot \mathrm{d}^{-1}$, e obtida a partir da lei de Beer expressa pela Equação 2 (MONTEITH; UNSWORTH, 2013; VIANNA; MARIN; PILAU, 2017); Rue é constante e igual a 1,395 g.MJ-1, baseado em uma média dos trabalhos expressos na Tabela 1; o índice de colheita (IC) utilizado é igual a 0,475, também sendo uma média baseada nos valores da Tabela 1 ; u é a umidade de grão, igual a 0,13 (DE ALENCAR et al., 2009).

$$
\operatorname{aPAR}=(1-\alpha) \cdot \operatorname{PAR} \cdot\left(1-e^{-k \cdot \operatorname{IAF}}\right)
$$

Sendo $\alpha=0,18$, o albedo médio para a cultura da soja; $\mathrm{k}$ o coeficiente de extinção, adotado constante nesse caso, igual a 0,55 (VIANNA; MARIN; PILAU, 2017).

O IAF varia ao longo do ciclo, assim foi realizada uma calibração para estimar o IAF diário em função da radiação aPAR (Figura 1). Além do IAF, dois outros parâmetros variaram diariamente, a função da temperatura média do ar (Ft) (Figura 2) e a função em relação ao fotoperíodo (Fcd).

A função de temperatura pondera o desenvolvimento da planta em relação à temperatura média do ar, ou seja, quanto mais próximo do intervalo ótimo a temperatura do ar estiver maior será o desenvolvimento, e $\mathrm{F}_{\mathrm{t}}$ tenderá a um (BOOTE et al., 1998). A temperaturas cardinais utilizadas foram: temperatura basal inferior $(\mathrm{Tb})$ foi de $7^{\circ} \mathrm{C}$; temperatura basal superior (TB) foi de $38^{\circ} \mathrm{C}$; e as duas temperaturas ótimas foram $26^{\circ} \mathrm{C}$ e $30^{\circ} \mathrm{C}$ (NORMAN, 1978).

Com o mesmo conceito, o fotoperíodo também é ponderado em relação a valores cardinais referentes a cada cultivar (Equação 3):

$$
\mathrm{Fcd}=\frac{(N-\mathrm{Cdc})}{(\mathrm{Cdm}-\mathrm{Cdc})}
$$

sendo $\mathrm{N}$ o fotoperíodo do dia, Cdc o comprimento mínimo do dia (horas) para o desenvolvimento e Cdm é o comprimento mínimo do dia para que ocorra desenvolvimento ótimo. Exceto N, que varia em função da latitude e da declinação solar, $\mathrm{Cdc}$ e $\mathrm{Cdm}$ são constantes, sendo adotados os seguintes valores: Cdc igual à 15 h e Cdm igual 12,58 horas. 


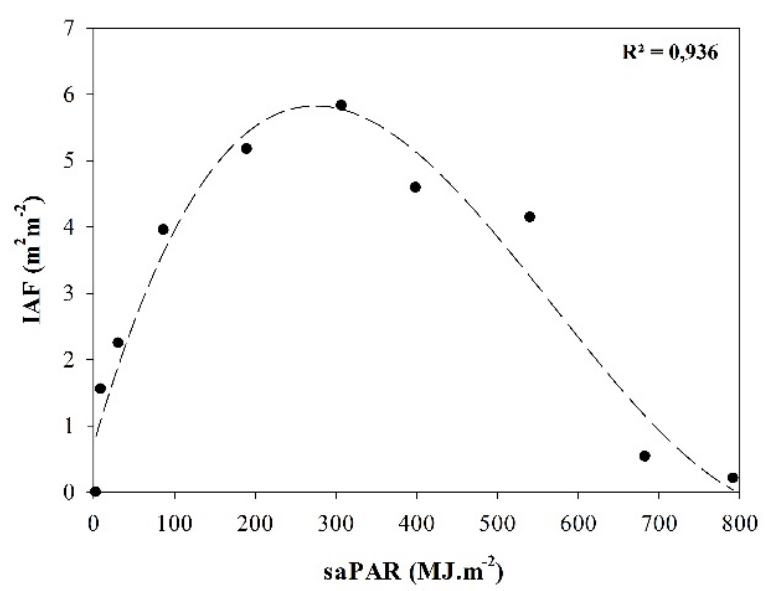

Figura 1. Curva de calibração entre o índice de área foliar (IAF) e o acumulado de radiação aPAR.

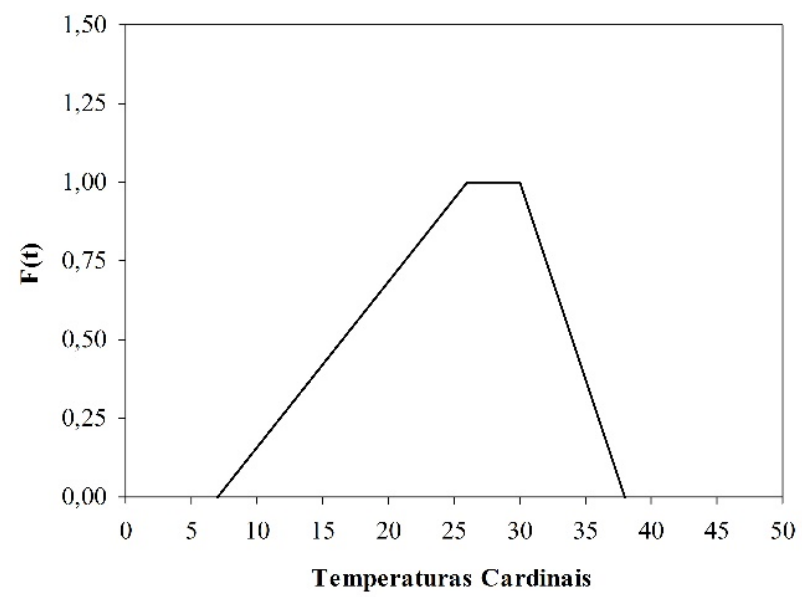

Figura 2. Comportamento da função de temperatura em relação as temperaturas cardinais da soja. Quanto temperatura do ar próximo a faixa ótima $\mathrm{F}(\mathrm{t})=1$.
Tabela 1. Constantes referentes a eficiência do uso da radiação (RUE) e índice de colheita (IC) para a cultura da soja.

\begin{tabular}{|c|c|c|c|}
\hline $\begin{array}{c}\text { RUE } \\
{[\text { g.MJ }}\end{array}$ & Referência & IC [\%] & Referência \\
\hline $0,87-1,31$ & (ADEBOYE et al., 2016) & $45-63$ & (ADEBOYE et al., 2016) \\
\hline $0,97-1,92$ & $\begin{array}{c}\text { (CONFALONE; DUJMOVICH, } \\
1999 \text { ) }\end{array}$ & $31-42$ & (DAVID et al., 2009) \\
\hline 0,88 & $\begin{array}{l}\text { (MUCHOW; ROBERTSON; } \\
\text { PENGELLY, 1993) }\end{array}$ & $50-64$ & (SPAETH et al., 1984) \\
\hline
\end{tabular}

\section{Indicadores estatísticos de eficiência de modelagem}

Os dados de entrada foram comparados estatisticamente aos simulados pelo modelo DSSAT/CROPGRO Soybean. Para tal, aplicou-se os testes estatísticos, $\mathrm{R}^{2}$ (Equação 4), DMA (desvio médio absoluto, Equação 5), REQM (raiz do erro quadrático médio, Equação 6) e índice de concordância D-Wilmott (Equação 7). Ressalta-se que tais testes foram aplicados apenas para as variáveis vegetativas, pois a variável de produtividade final foi coletada uma única vez (no final do ciclo da cultura).

$$
\mathrm{R}^{2}=\frac{\sum_{i=1}^{n}\left(E_{i}-\bar{O}\right)^{2}}{\sum_{i=1}^{n}\left(E_{i}-\bar{O}\right)^{2}+\sum_{i=1}^{n}\left(E_{i}-O_{i}\right)^{2}}
$$

em que $\mathrm{R}^{2}$ é o coeficiente de determinação, o valor observado, o valor simulado e a média dos valores observados.

$$
\mathrm{DMA}=\frac{\sum_{i=1}^{n}\left|e_{t}\right|}{n}
$$

em que DMA é o desvio médio absoluto, a diferença do valor observado e o simulado e n o número de períodos.

$$
\mathrm{RQME}=\sqrt{\frac{\sum(\mathrm{Ei}-\mathrm{Oi})^{2}}{n}}
$$

em que RQME é a raiz do erro quadrático médio, $\mathrm{O}_{i}$ o valor observado, $\mathrm{E}_{\mathrm{i}}$ a média dos valores observados $\mathrm{n}$ o número de períodos.

$$
D=1-\left[\frac{\sum_{i=1}^{n}\left(M_{i}-E_{i}\right)^{2}}{\sum_{i=1}^{n}\left(\left|M_{i}-\bar{M}\right|+\left|E_{i}-\bar{M}\right|\right)^{2}}\right]
$$

em que D é o índice de concordância de Willmott (1982), $\mathrm{M}_{\mathrm{i}}$ é o valor medido de ordem $\mathrm{i}, \mathrm{E}_{\mathrm{i}}$ é o valor estimado de ordem i, é a média dos valores medidos, e n o número dos valores medidos.

\section{Cenários de mudanças climáticas}

As projeções de clima futuro são oriundas de três Modelos de Clima Global (MCGs) (Tabela 2), participantes do Coupled Models Intercomparison Project Phase 5 (CMIP5), fornecidas pelo Program for Climate Model Diagnosis and Intercomparison e disponibilizado pelo Earth System Grid Data Portal (TAYLOR; STOUFFER; MEEHL, 2012). No entanto, optou-se pela utilização dos modelos globais do Intergovernmental Panel on Climate Change (IPCC) primeiramente pela indisponibilidade de resultados atualizados de modelos regionais (os quais são altamente custosos computacionalmente), e pelo fato da nova geração de modelos presentes no quinto relatório do IPCC (IPCC AR5) representarem o estado da arte em modelagem do sistema climático. 
Tabela 2. Resolução e origem dos Modelos de Clima Global utilizados.

\begin{tabular}{ccc}
\hline Modelos & Resolução (lat/lon) & Referência \\
\hline IPSL-CM5A-LR & $1.9^{\circ} \times 3.8^{\circ}$ & (DUFRESNE et al., 2013) \\
MRI-CGCM3 & $1.0^{\circ} \times 0.5^{\circ}$ & (YUKIMOTO et al., 2012) \\
MIROC5 & $1.4^{\circ} \times 1.4^{\circ}$ & (WATANABE et al., 2010) \\
\hline
\end{tabular}

Tabela 3. Principais características dos Representative Concentration Pathways (RCPs); adaptado de Moss et al., (2010).

\begin{tabular}{|c|c|c|}
\hline Nome/Cenário & Forçante Radiativa & $\begin{array}{c}\text { Concentração } \mathrm{CO}_{2} \\
\text { (p.p.m) }\end{array}$ \\
\hline RCP 4.5/Intermediário & $\begin{array}{c}\sim 4.5 \text { w.m }{ }^{-2} \text { e estabilização } \\
\text { depois de } 2100\end{array}$ & $\begin{array}{c}650 \mathrm{CO}_{2} \text { equivalente } \mathrm{e} \\
\text { estabilização depois de } \\
2100\end{array}$ \\
\hline RCP 8.5/Pessimista & $>8.5$ w.m $\mathrm{m}^{-2} \mathrm{em} 2100$ & $\begin{array}{c}>1370 \mathrm{CO}_{2} \text { equivalente } \\
\text { em } 2100\end{array}$ \\
\hline
\end{tabular}

Tabela 4. Incremento de temperatura do ar para os cenários de projeção, P1 (2020-2049) e P2 (2050-2080).

\begin{tabular}{lcc}
\cline { 2 - 3 } & P1 & P2 \\
\hline RCP 4.5 & $0,73^{\circ} \mathrm{C}$ & $1,34^{\circ} \mathrm{C}$ \\
RCP 8.5 & $1,15^{\circ} \mathrm{C}$ & $2,56^{\circ} \mathrm{C}$ \\
\hline
\end{tabular}

Tabela 5. Indicadores estatísticos de eficiência de modelagem.

\begin{tabular}{lcccc}
\hline Variável de Entrada & $\mathbf{R}^{\mathbf{2}}$ & DMA & RQME & D \\
\hline IAF & 0.94 & 0.3 & 0.40 & 0.97 \\
Biomassa de folhas & 0.95 & 102.0 & 122.43 & 0.98 \\
Biomassa de caule & 0.98 & 142.0 & 166.91 & 0.99 \\
Biomassa vegetativa & 0.97 & 235.0 & 265.80 & 0.99 \\
Área foliar específica & 0.12 & 53.5 & 71.58 & 0.60 \\
\hline
\end{tabular}

As simulações climáticas futuras do CMIP5 são realizadas utilizando-se a nova geração de cenários de forçantes radiativas Representative Concentration Pathways (RCP) (MOSS et al., 2010). Neste trabalho foram utilizados os RCPs 4.5 e 8.5 para abranger um cenário mais próximo ao presente, RCP 4.5, e o cenário mais pessimista, RCP 8.5.

As projeções foram separadas em dois períodos para obtenção da variação de temperatura média do ar. Tais períodos foram, 2020 a 2050 (P1) e 2050 a 2080 (P2), onde foram comparados com um intervalo de 30 anos do cenário historical, também simulado pelos MGC, no período de 1971 a 2000 para obtenção do viés de cada projeção. Assim obteve-se quatro valores de incremento de temperatura, os quais foram adicionados aos valores registrados de temperatura do ar e consequentemente inseridos nos modelos de produtividade.

\section{Resultados e discussão}

Os valores dos indicadores estatísticos obtidos para a correlação dos dados observados em função da curva de simulação obtida pelo modelo DSSAT/CROPGRO Soybean são apresentados na Tabela 5. Nota-se uma boa acurácia e precisão da simulação para as variáveis IAF, biomassa de folhas, biomassa de caule, biomassa vegetativa e uma baixa precisão e acurácia da variável área foliar específica.

A obtenção da calibração do modelo foi obtida pelo ajuste dos parâmetros pós a obtenção de uma boa calibração das variáveis vegetativas do modelo, com o ajuste dos parâmetros CSDL, EM-FL e SD-PM. E os parâmetros WTPSD, LFMAX, SLAVR foram ajustados para a calibração da produtividade. Nota-se que não há análise estatística para o dado de produtividade, pois foi realizada uma única cole- 
Tabela 6. Valores de produtividade potencial total, em t.ha-1, para todos os cenários avaliados, atual e futuro. Considerando os dois modelos, MAGé e DSSAT, sendo o segundo para duas possibilidades: sem incremento de $\mathrm{CO}_{2}$ (DSSAT- $\mathrm{CO}_{2}$ ) e com incremento de $\mathrm{CO}_{2}\left(\right.$ DSSAT-s $\left./ \mathrm{CO}_{2}\right)$.

\begin{tabular}{|c|c|c|c|c|c|}
\hline \multicolumn{6}{|c|}{ Produtividade Potencial (t.ha $\left.{ }^{-1}\right)$} \\
\hline \multirow{2}{*}{ Modelos } & \multirow{2}{*}{ Atual } & \multicolumn{2}{|c|}{ P1 } & \multicolumn{2}{|c|}{$\mathbf{P 2}$} \\
\hline & & RCP4.5 & RCP8.5 & RCP4.5 & RCP8.5 \\
\hline DSSAT-CO ${ }_{2}$ & 4,173 & 5,666 & 6,055 & 5,552 & 5,874 \\
\hline DSSAT-s/CO ${ }_{2}$ & 4,173 & 3,972 & 3,933 & 3,824 & 3,468 \\
\hline MAGé & 6.530 & 6,856 & 7,044 & 7,129 & 7,642 \\
\hline
\end{tabular}
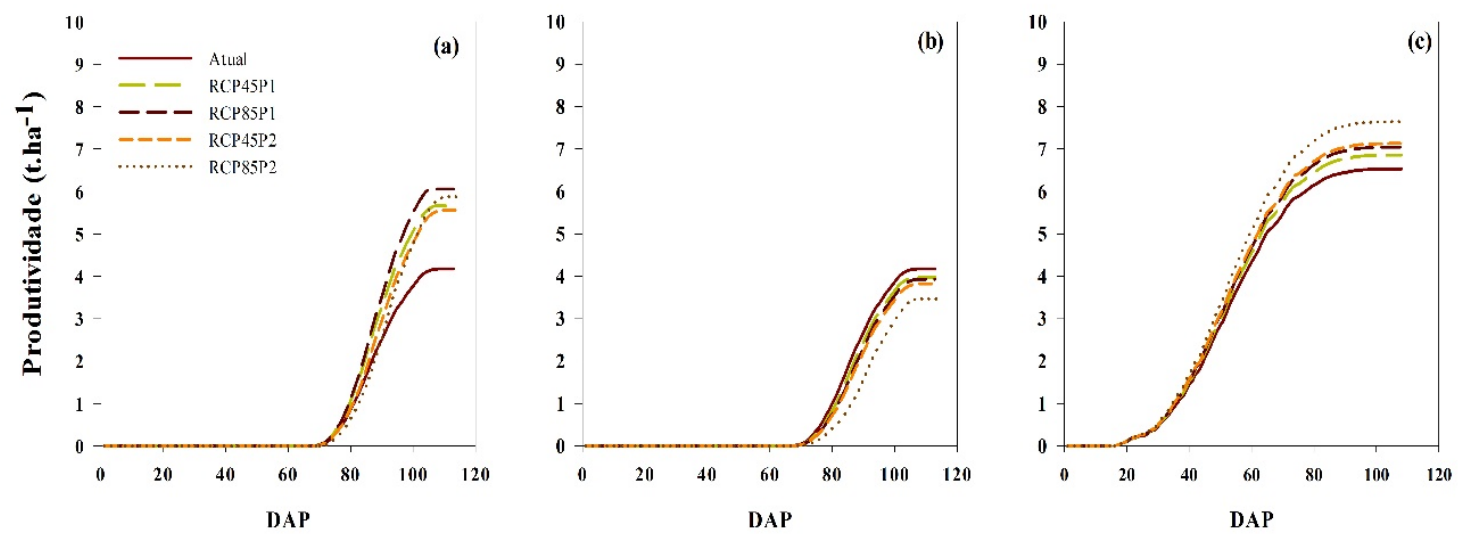

Figura 3. Variação da produtividade ao longo do ciclo. (a) Produtividade pelo DSSAT, considerando a concentração de CO2; (b) Produtividade estimada pelo DSSAT sem considerar a concentração de CO2; (c) Produtividade estimada pelo modelo MAGé adaptado.

ta e, portanto um único ponto. A produtividade final obtida, em massa seca, foi de $4.250 \mathrm{~kg} \cdot \mathrm{ha}^{-1}$ enquanto a simulada foi $4.173 \mathrm{~kg} \cdot \mathrm{ha}^{-1}$.

No modelo já calibrado para a região de Piracicaba introduzido incrementos de temperatura baseado nos modelos de mudanças climáticas. Na Figura 3b, observa-se uma gradativa queda de produtividade na região de Piracicaba, a medida que ocorre os incrementos de temperatura. Para os cenários RCP 4.5 (2020-2050), RCP 8.5 (2020-2050), RCP 4.5 (2050-2080), RCP 8.5 (2050-2080), obteve-se perdas de produtividade, respectivamente, em termos percentuais, de $-4,82,-5,75,-8,36$ e $-16,9$. Tais simulações seguiram os resultados de queda de produtividade obtidos pelo estudo de Assad et al (2008), tal estudo considerava os cenários A2 e B2 e apenas o incremento de temperatura. No referido estudo as perdas de produção brasileira chegariam a $40 \%$ no cenário mais pessimista.

Na Figura 3a, considera-se o efeito do aumento da concentração de dióxido de carbono $\left(\mathrm{CO}_{2}\right)$. Sabe-se que o aquecimento global ocorrerá concomitantemente com o aumento da concentração de $\mathrm{CO}_{2}$ atmosférico. $\mathrm{O}$ modelo DSSAT/CROPGRO Soybean é capaz de simular o incremento de $\mathrm{CO}_{2}$ sob a ótica da fisiologia vegetal da planta de soja. Uma vez que, $\mathrm{O} \mathrm{CO}_{2}$ é substrato primário da fotossíntese e pode beneficiar mais as plantas de metabolismo C3, como a soja, comparativamente às plantas C4 (KIMBALL, 1983; TAIZ e ZEIGER, 1991). Alguns estudos envolvendo a utilização de modelos de crescimento de culturas já consideram tais variáveis (PINTO, 2013; MARIN, 2014; BATTISTI, 2016) e podem contribuir com projeções de oferta de soja ao longo do tempo. Observa-se na Figura 3a, que os efeitos da mudança climática passam a ser benéficos em todos os cenários testados. Para os cenários RCP 4.5 (2020-2050), RCP 8.5 (2020-2050), RCP 4.5 (2050-2080), RCP 8.5 (2050-2080), obteve-se incrementos de produtividade, respectivamente, em termos percentuais, de 35,78, 45,09, $33,05,40,76$. Nessas perspectivas os cenários de mudanças climáticas beneficiariam a produção de soja na região de Piracicaba.

Buscando entender como o modelo DSSAT/CROPGRO explora a questão do $\mathrm{CO}_{2}$ no desenvolvimento da planta, recorreu-se ao código da programação. $\mathrm{O} \mathrm{CO}_{2}$ aparece como um fator de ajuste no processo fotossintético, em que o efeito do aumento da concentração $\mathrm{CO}_{2}$ pode ser incluído na rotina relacionada aos processos fotossintéticos do modelo isso é demonstrado nas Equações 8, 9 e 10.

$$
C C K=\text { CCEFF } / \text { CCMAX }
$$

em que CCK é a razão que representa a relação entre o $\mathrm{CO}_{2}$ e a fotossíntese do dossel, CCEFF é a eficiência relativa da assimilação de $\mathrm{CO}_{2}$ e CCMAX é o valor máximo de fotossíntese diária obtida a uma concentração de $330 \mathrm{vpm}$ de $\mathrm{CO}_{2}$. 


$$
\mathrm{A} 0=-\mathrm{CCMAX} *(1 .-\operatorname{EXP}(-\mathrm{CCK} * \mathrm{CCMP}))
$$

em que A0 é o efeito de referência do $\mathrm{CO}_{2}$ a uma concentração de $330 \mathrm{vpm}, \mathrm{CCMP}$ é o ponto de compensação de $\mathrm{CO}_{2}$.

$$
\text { PRATIO }=\text { A0 }+C C C M A X *(1 .-\operatorname{EXP}(-C C K * \text { CO2 }))
$$

em que PRATIO é o efeito relativo da concentração de $\mathrm{CO}_{2}$ na fotossíntese.

Já o fator temperatura é empregado de maneira mais complexa no modelo, em diversas sub-rotinas, como: na partição de carboidratos, lipídios, proteínas; penalização da produção pelo efeito de estresse e duração das fases fenológicas da cultura. Expressar todas as equações envolvidas deixaria o presente estudo excessivamente longo e complexo.

O modelo MAGé adaptado superestimou os valores de produtividade potencial no período presente, em aproximadamente 2 t.ha ${ }^{-1}$. Esse resultado é justificável, uma vez que o modelo é simplista e não considera parâmetros relacionado principalmente ao solo. Além disso, neste trabalho os valores de RUE, IC e $\mathrm{k}$ foram previamente definidos como constantes em todo o ciclo da cultura, sendo que neste mesmo trabalho encontramos os valores de RUE e IC variando de 0,87 a 1,92 e 0,31 a 0,64, respectivamente, como mostrado anteriormente na Tabela 1.

Ao longo do ciclo, Figura 3c, nota-se que a produtividade tem um crescimento mais acelerado que o modelo DSSAT em suas duas condições. Mas o MAGé, mesmo superestimando os valores de produtividade conseguiu acertar a curva de produtividade, sendo possível melhorar o modelo com calibrações adequadas.

Em cenários futuros, o modelo MAGé, como esperado apresentou um aumento de produtividade, mesmo sem o incremento de $\mathrm{CO}_{2}$ utilizado no DSSAT. Esse aumento é explicado pois com o aumento da temperatura do ar, os valores de temperatura média irão ficar cada vez mais dentro do limiar ótimo da cultura da soja (temperatura do ar entre as temperaturas ótimas da soja). Consequentemente, a função que pondera o desenvolvimento em relação a temperatura do ar será mais vezes próximo a 1, fazendo com que a produtividade seja cada vez mais dependente da radiação PAR.

\section{Conclusões}

O DSSAT/CROPGRO Soybean simulou de maneira satisfatória a produção atual de soja na região de Piracicaba, em relação aos dados obtidos em campo. Em contexto de mudanças climáticas, observou-se uma queda da produtividade ao considerar apenas o aumento da temperatura. Ao adicionar o incremento na $\left[\mathrm{CO}_{2}\right]$ observou-se um aumento na produtividade de soja na região. Portanto, os efeitos das mudanças climáticas contribuiriam para um aumento da produtividade de soja na região estudada.

A adaptação do modelo MAGé tendeu a superestimar a produtividade atual de soja em relação aos dados obtidos em campo. Notou-se que o modelo apresentou incremento na produtividade de soja, em todos os cenários de mudanças climáticas, considerando apenas o aumento da temperatura. Tal resultado divergiu do encontrado pelo DSSAT/ CROPGRO.

\section{Agradecimentos}

À Fundação de Amparo à Pesquisa do Estado de São Paulo (FAPESP), processo 2015/25702-3, pela bolsa de Mestrado e reserva técnica concedidas. Ao Grupo de Pesquisa e Experimentação em Modelagem Agrícola (GEPEMA), por todo o apoio na condução desse experimento e ao Departamento de Engenharia de Biossistemas da ESALQ/USP pela infraestrutura para realização desta pesquisa.

\section{Referências}

ADEBOYE, O. B. et al. Impact of water stress on radiation interception and radiation use efficiency of Soybeans (Glycine max L. Merr.) in Nigeria. Brazilian Journal of Science and Technology, v. 3, n. 1, p. 15 , 4 dez. 2016.

ALPHA, C. C.; CHIANG, A. C. Matemática para economistas. São Paulo: São Paulo Pearson Makron Books, 2005.

ASSAD, E. D. et al. Aquecimento global e a nova geografia da produção agrícola no Brasil. Embrapa-Cepagri, São Paulo, 2008.

BATTISTI, Rafael. Calibration, uncertainties and use of soybean crop simulation models for evaluating strategies to mitigate the effects of climate change in Southern Brazil. Tese de Doutorado. Universidade de São Paulo.

BOOTE, K. J. et al. The CROPGRO model for grain legumes. In: Understanding options for agricultural production. [s.l.] Springer, 1998. p. 99-128.

CONFALONE, A.; DUJMOVICH, N. Influência do "déficit" hídrico sobre a eficiência da radiação solar em soja. Revista Brasileira de Agrociência, v. 5, n. 3, p. 195-198, 1999.

CONAB: COMPANHIA NACIONAL DE ABASTECIMENTO.

Acompanhamento de safra brasileira de grãos, Segundo Levantamento, Brasília, novembro/2016. Disponível em: <http:// www.conab.gov.br/conteudos.php?a=1252\&t=2\&P agina_ objcmsconteudos=3\#A_objcmsconteudos>. Acesso em: 11 jan. 2017.

DAVID, M. B. et al. Modeling denitrification in a tile-drained, corn and soybean agroecosystem of Illinois, USA. Biogeochemistry, v. 93, n. 1-2, p. 7-30, 2 mar. 2009.

DE ALENCAR, E. R. et al. Qualidade dos grãos de soja armazenados em diferentes condições. Revista Brasileira de Engenharia Agrícola e Ambiental, v. 13, n. 5, p. 606-613, 2009.

DUFRESNE, J.-L. et al. Climate change projections using the IPSL-CM5 Earth System Model: from CMIP3 to CMIP5. Climate Dynamics, v. 40, n. 9-10, p. 2123-2165, 2013.

EMBRAPA: EMPRESA BRASILEIRA DE PESQUISA AGROPECUÁRIA. Soja - BRS 399RR, 2016. Disponível em: <https://www.embrapa.br/soja/ busca-de-produtos-processos-e-servicos/-/produto-servico/2919/soja--brs-399rr>. Acesso em: 14 maio. 2017

FOLEY, J. A. CAN WE FEED THE WORLD \& SUSTAIN THE PLANET? Scientific American, v. 305, n. 5, p. 60-65, 2011. ISSN 00368733. 
Disponível em: < http://search.ebscohost.com/login.aspx?direct=true\&d $\mathrm{b}=$ aph\&AN=66913738[=pt-br\&site=ehost-live $>$.

JONES, J. W.; KEATING, B. A.; PORTER, C. H. Approaches to modular model development. Agricultural Systems, v. 70, n. 2, p. 421-443, 2001

KERBAUY, G. Fisiologia Vegetal-2004: Editora Guanabara Koogan/RJ.

KIMBALL, B. A. Carbon dioxide and agricultural yield: an assemblage and analysis of 430 prior observations. Agronomy Journal, v. 75, n. 5, p. 779788, 1983. ISSN 0002-1962.

LINCOLN, T.; TAIZ, L. Plant physiology. Redwood City, Calif: Redwood City, Calif. Benjamin/Cummings Pub. Co., 1991.

MARIN, F. R. Eficiência de produção da cana-de-açúcar brasileira estado atual e cenários futuros baseados em simulações multimodelos. 2014. Piracicaba.

MATTOS, E. C. et al. Estudo da identidade histológica de subprodutos de soja (Glycine max L.). Revista do Instituto Adolfo Lutz, v. 74, n. 2, p. 104-110, 2016. ISSN 1983-3814.

MONTEITH, J.; UNSWORTH, M. Principles of environmental physics: plants, animals, and the atmosphere. [s.l.] Academic Press, 2013.

MOSS, R. H. et al. The next generation of scenarios for climate change research and assessment. Nature, v. 463, n. 7282, p. 747-756, $11 \mathrm{fev}$. 2010.

MUCHOW, R. C.; ROBERTSON, M. J.; PENGELLY, B. C. Radiationuse efficiency of soybean, mugbean and cowpea under different environmental conditions. Field Crops Research, v. 32, n. 1-2, p. 1-16, fev. 1993

NORMAN, A. G. 1 - Background. In: Soybean Physiology, Agronomy, and Utilization. [s.l: s.n.]. p. 1-15.

PINTO, H. M. S. Projeções de risco de produção de cana-de-açúcar no Estado de São Paulo baseadas em simulações multimodelos e cenários climáticos futuros. 2015. Piracicaba.
POPPER, K. The logic of scientific discovery. Routledge, 2005. ISBN 1134470029 .

ROSENZWEIG, C. et al. Assessing agricultural risks of climate change in the 21st century in a global gridded crop model intercomparison. Proceedings of the National Academy of Sciences, v. 111, n. 9, p. 32683273, 2014. ISSN 0027-8424.

SILVA, J. F. D. Inter-relações entre produção e demanda da soja e de proteína animal. 2014.

SPAETH, S. C. et al. Stability of Soybean Harvest Index1. Agronomy Journal, v. 76, p. 482-486, 1984

TAYLOR, K. E.; STOUFFER, R. J.; MEEHL, G. A. An overview of CMIP5 and the experiment design. Bulletin of the American Meteorological Society, v. 93, n. 4, p. 485-498, 2012.

USDA: United States Department of Agriculture. World agricultural supply and demand estimates. Washington (DC), 2017. Disponível em: <http://www.usda.gov/oce/commodity/wasde/latest.pdf>. Acesso em: 02 jan 2017

VIANNA, M. S.; MARIN, F. R.; PILAU, F. G. Modelo agrometeorológico genérico de produção. [s.l: s.n.].

WATANABE, M. et al. Improved climate simulation by MIROC5: mean states, variability, and climate sensitivity. Journal of Climate, v. 23, n. 23, p. 6312-6335, 2010.

YUKIMOTO, S. et al. A new global climate model of the Meteorological Research Institute: MRI-CGCM3-model description and basic performance- . Journal of the Meteorological Society of Japan. Ser. II, v. 90, p. 23-64, 2012.

REFERENCIAÇÃO

SILVA, E. H. F. M. da; PEREIRA, R. A. de A.; GONÇALVES, A. O.; BORDIGNON, Á. J. Z.; MARIN, F. R. Simulação de produtividade futura de soja em Piracicaba-SP com base em projeções de mudanças climáticas. Agrometeoros, Passo Fundo, v.25, n.1, p.9-17, 2017.

Declaração: os trabalhos estão sendo publicados nesse número de AGROMETEOROS (v.25, n.1, ago 2017) conforme foram aceitos pelo XX Congresso Brasileiro de Agrometeorologia, realizado de 14 a 18 de agosto de 2017, em Juazeiro, BA e Petrolina, PE, sem revisão editorial adicional da revista. 


\section{Simulation of soybean yield in Piracicaba-sp based on climate change}

Evandro Henrique Figueiredo Moura da Silva ${ }^{1}$, Rodolfo Armando de Almeida Pereira ${ }^{2}$, Alexandre Ortega Gonçalves ${ }^{3}$, Álex Júnior Zanchet Bordignon ${ }^{4}$, Fábio Ricardo $\operatorname{Marin}^{5(*)}$

${ }^{1}$ Mestrando em Engenharia de Sistemas Agrícolas, ESALQ/USP, Piracicaba, São Paulo, ehfmsilva@usp.br

${ }^{2}$ Mestrando em Engenharia de Sistemas Agrícolas, ESALQ/USP, Piracicaba, São Paulo, rodolfopereira@usp.br

${ }^{3}$ Doutorando em Engenharia de Sistemas Agrícolas, ESALQ/USP, Piracicaba, São Paulo, alexandre.ortega@embrapa.br

${ }^{4}$ Graduando em Engenharia Agronômica, ESALQ/USP, Piracicaba, São Paulo, alex.bordignon@usp.br

${ }^{5}$ Professor Associado do Departamento de Engenharia de Biossistemas, ESALQ/USP, Piracicaba, São Paulo, fabio.marin@usp.br

${ }^{(*)}$ Autor para correspondência

\section{ARTICLE INFO}

Article history:

Received 16 June 2017

Accepted 10 August 2017

\section{Index terms:}

crop models

DSSAT

MAGé

Glycine max

\section{ABSTRACT}

Brazil is one of the largest soybean-producing countries (Glycine max L.). This plant is the main source of food protein on the planet. Based on the economic and social importance of soybeans in the world context, it is important to consider the future projections of soybean production in the international debate on climate change; And forecast the global population increase of 2 billion of people by the year 2050 . Thus, we intend to evaluate the impact of climate change on the development of soybean crop in the region of Piracicaba-SP using two models DSSAT / CROPGRO Soybean and MAGé adapted. The DSSAT/CROPGRO model was calibrated for the region of the study with data from climate, soil and biometric data of soybean (BRS 399RR) obtained experimentally. The IPCC climate change scenarios selected in this study were RCP4.5 (2020-2050), RCP4.5 (2020-2050), RPP8.5 (2050-2080) and RCP8.5 (2050-2080). It was observed that the DSSAT / CROPGRO model presented a good calibration, representing the current soy production in the region. Regarding the simulations in future scenarios, there was a decrease in soybean yield in the region when only the temperature factor was considered and productivity increase when the increase in $\left[\mathrm{CO}_{2}\right]$ was considered in the model. In this second perspective, climate change scenarios would be beneficial to soy production in Piracicaba. The adapted MAGé model showed overestimated productivities in all the studied situations.

\section{CITATION}

SILVA, E. H. F. M. da; PEREIRA, R. A. de A.; GONÇALVES, A. O.; BORDIGNON, Á. J. Z.; MARIN, F. R. Simulação de produtividade futura de soja em Piracicaba-SP com base em projeções de mudanças climáticas. Agrometeoros, Passo Fundo, v.25, n.1, p.9-17, 2017.

Disclaimer: papers are published in this issue of AGROMETEOROS (v. 25, n.1, aug 2017) as accepted by the XX Congresso Brasileiro de Agrometeorologia, held August 14-18, 2017 in Juazeiro, Bahia and Petrolina, Pernambuco, Brazil, without further revision by editorial board. 